Research Article

\title{
Thread-based microfluidic channels: Fabrication and application in organic semiconductor crystalline needles
}

\author{
Masashi Watanabe $^{1} \mathbb{D} \cdot$ Tsuyoshi Masuda $^{1}$
}

Received: 26 December 2019 / Accepted: 7 May 2020 / Published online: 12 May 2020

(c) Springer Nature Switzerland AG 2020

\begin{abstract}
Thread, which is a twisted bundle of monofilaments, can function as microfluidic channels. This application of thread can be attributed to the capillary action occurring in the narrow space between the monofilaments through which fluid can pass without the use of pumps. The microfluidic channels were fabricated by sandwiching thread between two glass slides. As expected, fluid spontaneously passed through the thread. However, the fluid was flooded into the space between the glass slides. This was also due to the capillary action because the contact angle of the fluid on the slides was smaller than $90^{\circ}$. This drawback was overcome by using a reservoir, a mechanism designed by considering the total surface free energy of the system. As an application of the channels made of thread, a method for the regular arrangement of crystalline needles of an organic semiconductor (9,10-dibromoanthracene) was developed. One of the glass slides sandwiching the thread was coated with polydimethylsiloxane, whose surface was oxidized through UV-ozone treatment. In the area of the polydimethylsiloxane surface adjacent to the thread, regular surface undulations were created by the organic solvents used as fluids. When dissolved in the solvent, the organic semiconductor precipitated as crystalline needles along the undulations. Because normal microfluidic channels are tunnels created in solid materials, only the space inside the channels can be used for applications. In contrast, the application developed in this study uses the space outside the channels, a novel methodology unique to thread-based channels.
\end{abstract}

Electronic supplementary material The online version of this article (https://doi.org/10.1007/s42452-020-2886-x) contains supplementary material, which is available to authorized users.

$\triangle$ Masashi Watanabe, mwatana@shinshu-u.ac.jp| ${ }^{1}$ Faculty of Textile Science and Technology, Shinshu University, 3-15-1 Tokida, Ueda, Nagano 386-8567, Japan. 


\section{Graphic abstract}

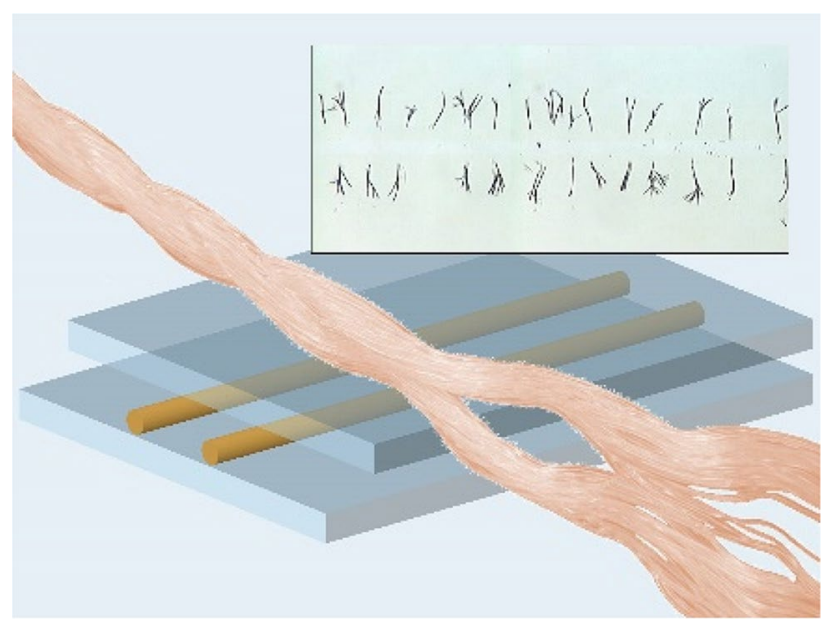

Keywords Thread · Microfluidic channels $\cdot$ Crystalline needles $\cdot$ Wrinkle $\cdot$ Buckling instability $\cdot$ Organic semiconductor

\section{Introduction}

Water can flow through a thread, which is a twisted bundle of monofilaments, because of the capillary action that occurs in the narrow spaces between the monofilaments [1,2]. Thread utilized for water transport has recently been recognized as a new class of channels in microfluidic devices $[3,4]$. The use of thread-based microfluidic channels has several potential advantages. Fluid passes through thread without the use of pumps, enabling threads to be used as channels in lowcost, portable diagnostic devices. Thread is easily bent and to some extent stretched, which are qualities desirable in flexible, wearable microfluidic devices. Thread can be prepared from a wide variety of materials, including polymers, metals, and carbon materials, suggesting the possibility of imparting such functionalities as separation, sensing, and conductivity. The surface area of a thread is much higher than that of the inner walls of same-diameter pipes that are normally used as microfluidic channels, making catalyst-coated thread potentially more effective in various catalytic organic syntheses. Therefore, we believe that the use of thread-based microfluidic channels should be investigated in detail. Some groups have recently proposed applications of thread-based microfluidic channels for diagnostics assays [5], chemical syntheses [6], and chemical analyses [7]. However, similar applications can also be realized using normal microfluidic channels and are not unique to thread-based microfluidic channels.

Normal microfluidic channels are tunnels created in solid materials, such as glass and polymers; only the space inside the channels is available for fluid flow, chemical syntheses, or analyses. In thread-based channels, the thread can contain fluids, even if not surrounded by solid materials. Therefore, we believe that applications using the space outside the channels are possible and will surely be unique to threadbased channels. In this study, we present a new method for producing a regular arrangement of crystalline needles outside of thread-based channels.

The regular arrangement of one-dimensional materials, at nanometer or micrometer scales, has been extensively investigated, and various methods for such arrangement have been proposed since the last two decades [8]. For example, organic semiconductor nanowires were regularly arranged using a dip-coating method to fabricate field-effect transistor arrays $[9,10]$. Moreover, microfluidic channels have been used as a method for the arrangement of nanowires [11], in which case, although the orientation of nanowires was well controlled, the spacing between nanowires was not. The control of both the orientation and the two-dimensional position of one-dimensional materials is still challenging [12]. In this study, we applied the thread-based channels to develop a method for the regular arrangement of organic semiconductor crystalline needles. We utilized the undulation of a substrate under the thread in order to control both the orientation and the position of the crystalline needles.

\section{Experiment}

\subsection{Untwisting of thread}

Twisted nylon thread (110 dtex $\times 1 \times 2)$ was purchased from KINKAME Shigyo Co., Ltd., Tokyo, Japan. As shown in 
Fig. 1a, the thread was divided into two thinner threads of $110 \mathrm{dtex}$; dtex" gives the weight in grams of a 10-kmlong thread. Each 110 dtex thread comprised 33 monofilaments of $20 \mu \mathrm{m}$ diameter. A glass slide was then suspended using the $110 \mathrm{dtex}$ thread (Fig. 1b). Because the slide was freely whirled, the excess torsion of the thread was removed. After suspending the slide for $30 \mathrm{~min}$, the thread was slightly stretched and exposed to water vapor at $100{ }^{\circ} \mathrm{C}$ for $1 \mathrm{~h}$. The thread was then dried at $100{ }^{\circ} \mathrm{C}$ for $2 \mathrm{~h}$, and straight, slightly twisted thread with a diameter of $\sim 0.16 \mathrm{~mm}$ was obtained (Fig. 1c).

\subsection{Oil-repellent treatment of glass slides}

On the glass sides (S-1215; Matsunami Glass Ind. Ltd., Osaka, Japan), whose surface was smooth or was roughened using an abrasive (Fuji white alumina WA220; Fuji Manufacturing Co. Ltd., Tokyo, Japan), $1 \mathrm{H}, 1 \mathrm{H}, 2 \mathrm{H}, 2 \mathrm{H}$-perfluorooctyltriethoxysilane (Sigma-Aldrich Japan Co. LLC, Tokyo, Japan) was spread. The slide was then placed in an oven at $100{ }^{\circ} \mathrm{C}$ for $2 \mathrm{~h}$ and subsequently rinsed with acetone.

\subsection{PDMS coating of glass slides}

The prepolymer (KE-103) of the polydimethylsiloxane (PDMS) elastomer and the curing agent (CAT-103) were obtained from Shin-Etsu Chemical Co. Ltd., Tokyo (Japan).

A

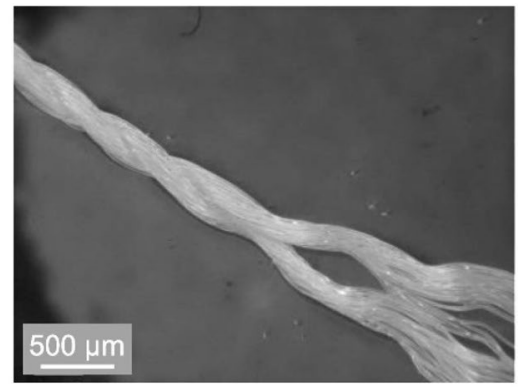

B

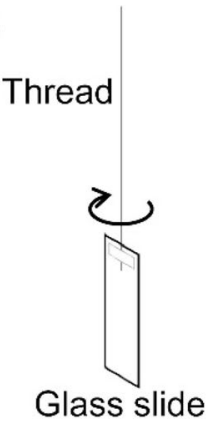

Fig. 1 Light microscope images of a twisted nylon thread and c slightly twisted, straight thread. b Method for removing excess torsion of the thread
A mixture of KE-103 and CAT-103 at a w/w ratio of 100:2 was degassed under vacuum, followed by casting onto a glass slide. The mixture was then vulcanized at $100{ }^{\circ} \mathrm{C}$ for $2 \mathrm{~h}$. The resultant PDMS elastomer was $\sim 0.5 \mathrm{~mm}$ thick. The PDMS surface was then oxidized using a UV-ozone cleaner (UV-TC-110; Bioforce Nanosciences, Ames, IO, USA) for $90 \mathrm{~min}$. Finally, the PDMS-coated glass slide was cut into 7-mm-wide and 18-mm-long rectangular pieces.

\subsection{Fabrication of microfluidic channels composed of thread}

The experimental setup consisted of thread, a base plate, top plate, cover plate, and glass weight (Fig. 2). The thread was used for causing fluid flow. The top plate was placed over the thread to construct a reservoir. The cover plate was also placed over the thread. In this paper, the word "channel" means the segment of thread under the cover plate. The glass weight $(\sim 5 \mathrm{~g})$ was placed on the cover plate to ensure complete contact between the thread and the cover plate. In some experiments, a Teflon sheet was additionally inserted under the base plate in order to conceal thread on the bottom of the base plate (Fig. S1 in the Electronic supplementary material).

The base plate was an oil-repellent glass slide, whose surface was either smooth or was roughened, depending on the experiments. The top plate was an oil-repellent or hydrophilic glass slide with a smooth surface. The cover

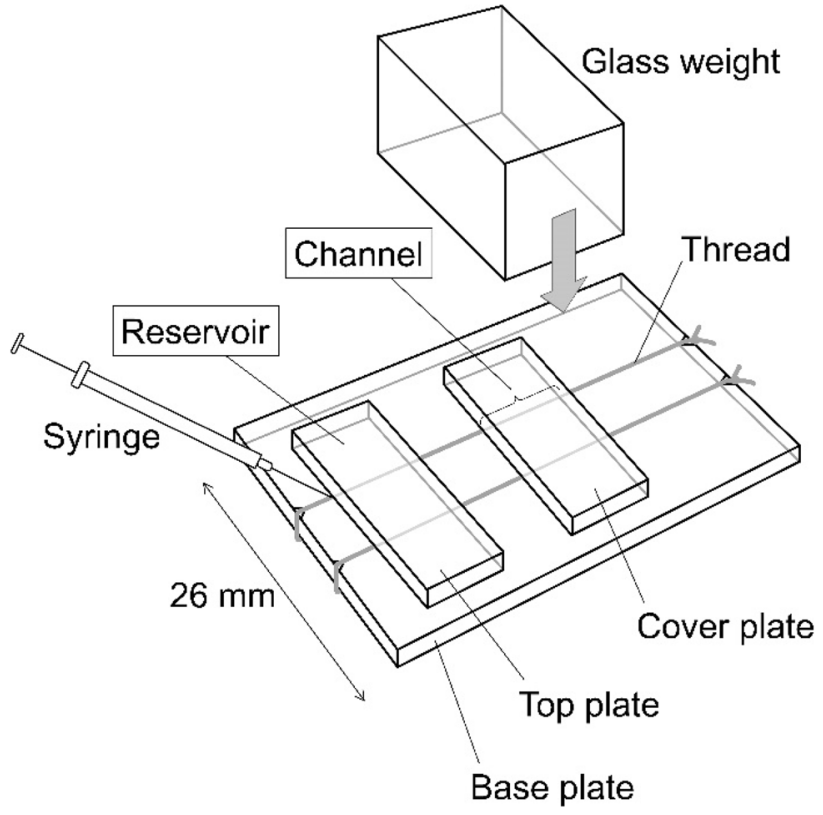

Fig. 2 Experimental setup of the channels made of thread 
plate was an oil-repellent, hydrophilic, or PDMS-coated glass slide. The experimental conditions are summarized in Table 1.

\subsection{Procedure for injecting fluid into channels}

In some experiments, prior to the injection of the fluid, the glass weight was pushed for $20 \mathrm{~s}$ using a force of 30 or $300 \mathrm{gf}$ to ensure contact between the thread and the cover plate (Table 1: force applied to the cover plate before injecting fluid). The fluid was then injected into the reservoir (i.e., the thin space between the top plate and the base plate) using a microsyringe (Fig. 2). The fluid spontaneously flowed into the channel due to the capillary action. When the cover plate was the PDMS-coated glass slide, the plate was flipped to observe its surface using a light microscope (MELUX-2-1 L; Kyowa Optical Co. Ltd., Kanagawa, Japan). Several light microscope images had to be combined, when a vast area was observed.

\subsection{Improved procedure for arranging crystalline needles}

This procedure was used for the regular arrangement of crystalline needles of 9,10-dibromoanthracene (DBA) on the PDMS-coated glass slide. As a pretreatment of the thread, the thread was wetted with the saturated solution of DBA dissolved in propyl acetate, followed by washing with acetone. This pretreatment was repeated thrice. As a pretreatment of the cover plate (the PDMS-coated glass slide), the plate was rinsed with methanol and dried by blowing air. After the cover plate and the glass weight were placed on the thread, the weight was lightly pushed using a force of $30 \mathrm{gf}$ for $20 \mathrm{~s}$. Another DBA solution was prepared by mixing $(a)$ pure ethyl acetate and $(b)$ a saturated solution of DBA in propyl acetate $(a: b=3: 2, w / w)$. This mixture $(1.3 \mu \mathrm{L})$ was injected into the reservoir. After $2 \mathrm{~min}$, the cover plate was flipped to observe its surface.

\subsection{Measurement of profile of surface undulation}

Profiles of the surface undulation were measured using a two-dimensional laser profiler (LT-9000; Keyence Corporation, Osaka, Japan).

\subsection{Measurement of contact angles}

A droplet $(1 \mu \mathrm{L})$ was placed on a substrate. Based on a photograph of the sideview of the droplet, the contact angle was measured.

\subsection{Scanning electron microscopy}

The surface of samples was observed using a scanning electron microscope (SEM) (SU1510; Hitachi High-Tech Corporation, Tokyo, Japan).

\section{Results and discussion}

\subsection{Injection of fluid into channels}

We planned to flow organic solvents through the channels made of thread in the applications (described later). Therefore, $\mathrm{N}$-methylpyrrolidone (NMP) was used as a model fluid, which contained a small amount of blue dye $(1 \% \mathrm{w} / \mathrm{w}$ acid blue 9). The capillary rise of NMP along a vertically suspended thread was measured to be $\sim 5 \mathrm{~cm}$, indicating that NMP would spontaneously flow through the channels.

Nylon thread was used in this study. Threads made from other materials, including cotton, silk, and polyester, could also cause movement of the fluid by capillary force. Polyester-cotton blended thread seemed unsuitable because it was fluffy.

Flow velocity of NMP along a horizontally placed nylon thread was evaluated using a wetted length of the thread, $L$. An example of the measured $L$ as a function of time $t$ is shown in Fig. S2 in the Electronic supplementary material. It

Table 1 Experimental conditions

\begin{tabular}{|c|c|c|c|c|c|}
\hline \multirow[t]{2}{*}{ No } & \multicolumn{3}{|l|}{ Surface properties } & \multirow{2}{*}{$\begin{array}{l}\text { Force applied to the cover } \\
\text { plate before injecting fluid }\end{array}$} & \multirow{2}{*}{$\begin{array}{l}\text { Volume of fluid } \\
\text { injected into the reser- } \\
\text { voir }(\mu \mathrm{L})\end{array}$} \\
\hline & Base plate & Top plate & Cover plate & & \\
\hline 1 & Oil-repellent, smooth & Non & Oil-repellent, smooth & Zero & 5 \\
\hline 2 & Oil-repellent, smooth & Oil-repellent, smooth & Oil-repellent, smooth & Zero & 4 \\
\hline 3 & Oil-repellent, smooth & Hydrophilic, smooth & Hydrophilic, smooth & Zero & 4 \\
\hline 4 & Oil-repellent, rough & Hydrophilic, smooth & PDMS-coated & $300 \mathrm{gf}$ & 2 \\
\hline 5 & Oil-repellent, smooth & Hydrophilic, smooth & PDMS-coated & $30 \mathrm{gf}$ & 2 \\
\hline 6 & Oil-repellent, rough & Hydrophilic, smooth & PDMS-coated & $30 \mathrm{gf}$ & 1.3 \\
\hline
\end{tabular}


was approximately proportional to the square root of time, as predicted by Washburn's equation $[2,13]$ :

$L=\sqrt{\frac{(\gamma \cos \theta) D t}{4 \mu}}$

where $\gamma$ is the surface tension, $\theta$ is the contact angle between the fluid and the thread surface, $D$ is the effective capillary diameter, and $\mu$ is the viscosity. Based on the average of four experiments, $L(\mathrm{~mm})$ and $t(\mathrm{~s})$ were expressed by the following equation:

$L=1.8 \sqrt{t}$

NMP was then injected into the channels by placing a droplet of NMP $(5 \mu \mathrm{L})$ on the thread. However, NMP was flooded out of the channel, as shown in Fig. 3a. Although both the base and cover plates were made of oil-repellent glass, the measured contact angle of NMP on the glass slide was $71^{\circ}$. This indicated that the droplet on the thread was sucked into the space between the base and cover plates due to the capillary action. Because contact angles of most organic solvents on the oil-repellent glass slide would be smaller than $90^{\circ}$, the flooding seemed to be an essential drawback of this setup.

To overcome this drawback, an additional plate (the "top plate" shown in Fig. 3b) was placed, and the space under this plate was used as a reservoir. The intended role of the reservoir is illustrated in Fig. 4. Let us assume that a certain amount of fluid was flooded, and the same volume of fluid was sucked from the reservoir. The total surface free energy would be unchanged, indicating that the flooding does not occur spontaneously. Therefore, we expected that flooding would be prevented by using the reservoir.

This hypothesis was confirmed by experiments using the oil-repellent cover plate (Fig. 3b). Based on the same mechanism, we expected that flooding would not
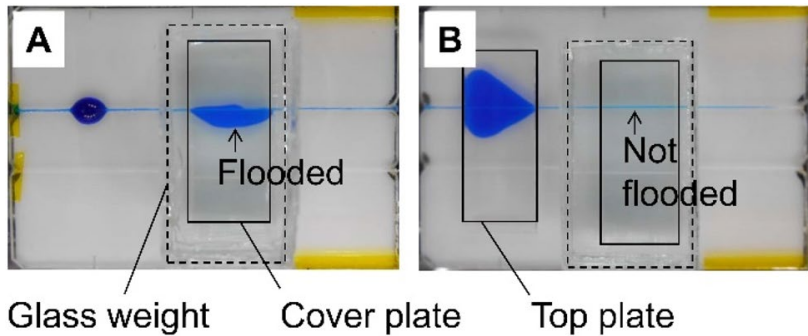

for reservoir

Fig. 3 Photographs of channels filled with NMP a when a droplet was simply placed on the thread (Experimental condition: No. 1) and $\mathbf{b}$ when fluid was supplied from the reservoir (Experimental condition: No. 2)

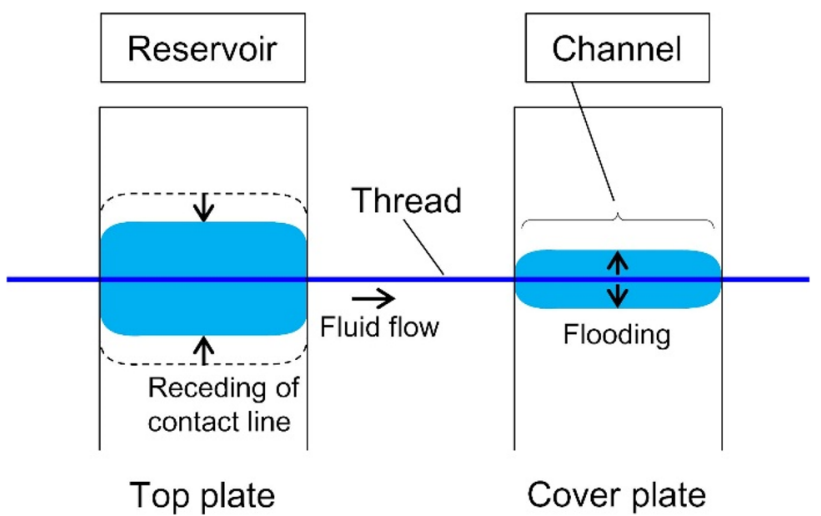

Fig. 4 Schematic illustration of the role of reservoir

occur even if the cover plate was hydrophilic, which was experimentally confirmed also (Fig. S3 in the Electronic supplementary material). We observed the channel for more than $15 \mathrm{~min}$ and confirmed that flooding did not occur, indicating that thread-based flow without flooding is possible when a reservoir is used.

\subsection{Regular arrangement of crystalline needles using the channels}

We intended to regularly arrange organic semiconductor crystalline needles using the channels made of thread. As an organic semiconductor, DBA was used because its crystalline needles are easily obtained by drying its solution, as reported by Tong et al. [9]. The cover plate was a PDMS-coated glass slide, whose surface was oxidized by the UV-ozone treatment. Such oxidized PDMS surfaces can be regularly undulated due to the buckling instability when exposed to appropriate organic solvents $[14,15]$. We expected the undulation to direct the crystallization of needles.

The channel was fabricated between the base plate with a rough surface and the PDMS-coated cover plate because the PDMS-coated plate easily sticks to the base plate when the surface of the base plate is smooth. The channel was filled with solutions in which DBA was dissolved in various solvents at saturated concentration for each. The cover plate was subsequently flipped to observe its surface. When the solvent was isoamyl acetate (IA), DBA crystalline needles were formed on the oxidized PDMS surface near the thread (Fig. 5). The crystalline needles were not embedded in the PDMS, which was confirmed by examining the surface profile using a two-dimensional laser profiler (Fig. S4 in the Electronic supplementary material). The crystalline needles were oriented roughly to the direction perpendicular to the thread. Similar results were obtained using the following solvents: methyl ethyl 


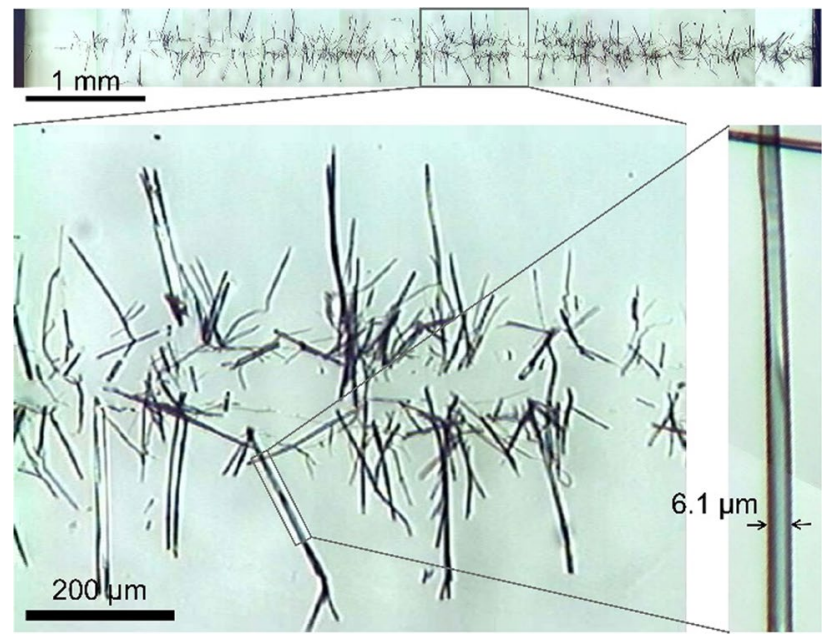

Fig. 5 Light microscope images of DBA crystalline needles formed after DBA solution flowed through the channel. The solvent was isoamyl acetate (Experimental condition: No. 4)

ketone (MEK), propyl acetate (PA), and diethylene glycol diethyl ether (DGDE). These solvents had contact angles smaller than $90^{\circ}$, even on the glass slide treated so as to be oil repellent using $1 \mathrm{H}, 1 \mathrm{H}, 2 \mathrm{H}, 2 \mathrm{H}$-perfluorooctyltriethoxysilane (Table $\mathrm{S} 1$ in the Electronic supplementary material).

The channel was continuously observed using a light microscope when the channel was filled with the solution (Fig. 6a) and when the solvent was evaporated (Fig. 6b). The formed undulation was perpendicular to the thread. Although the undulation was irrelevant to the position and orientation of the shorter crystalline needles, longer needles were mainly formed at the positions of the valleys of the undulation, suggesting that these needles grew in the valleys.

In a separate experiment, after a rectangular sample of the oxidized PDMS was dipped in IA, the profile of the resulting surface undulation was measured. As shown in Fig. S5 of the Electronic supplementary material, creases had formed with dimensions of $20 \pm 2 \mu \mathrm{m}$ in depth and $126 \pm 14 \mu \mathrm{m}$ in width, values obtained by finding the average depth and width of ten creases. The DBA crystalline needles seen on both sides of the thread may have formed in these creases where the solution dried to precipitate the crystals.

A simple model for the formation of crystalline needles is shown in Fig. 7. If a crystalline needle is formed by drying the solution filling a crease of $20 \mu \mathrm{m}$ depth and $126 \mu \mathrm{m}$ width (Fig. 7b), a crystalline needle of $2.7 \mu \mathrm{m}$ thickness is expected to be formed, based on the concentration (c) of the saturated solution of DBA in IA $(\sim 0.45 \%, w / w)$, the depth $(d)$, and the width $(w)$ of the crease using the following equation,
A

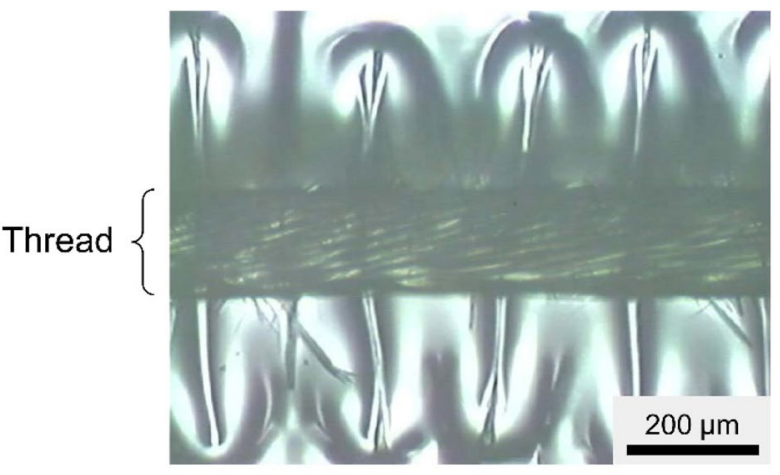

B

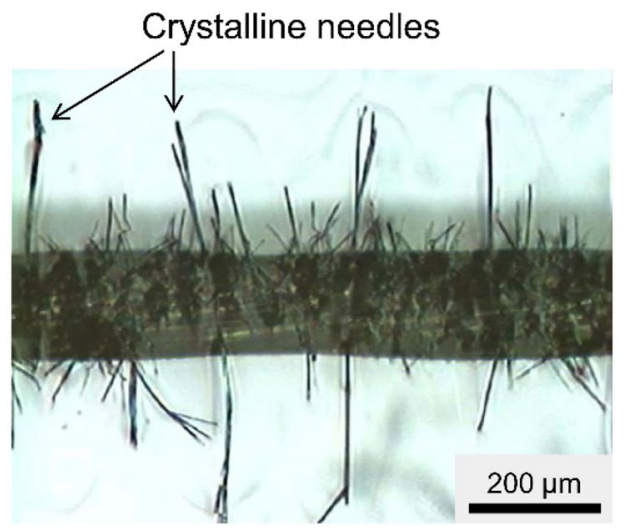

Fig. 6 a Undulation of the PDMS-coated cover plate surface, while the channel was filled with DBA solution. The solvent was isoamyl acetate. $\mathbf{b}$ Crystalline needles formed along the undulation after the evaporation of the solvent (Experimental condition: No. 5)

$\frac{d w c}{200}=\pi r^{2}$

where $r$ is the radius of the cross section of the crystalline needle. However, the observed crystalline needles were

A

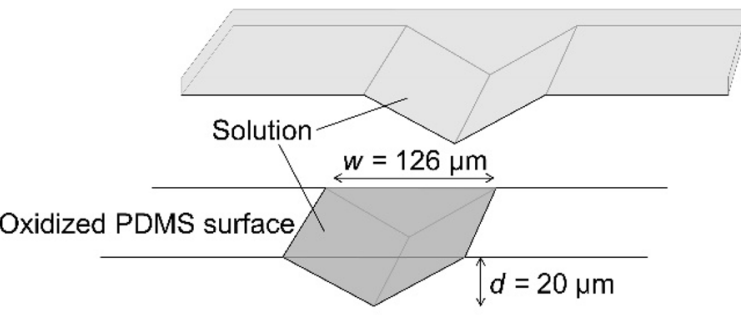

C

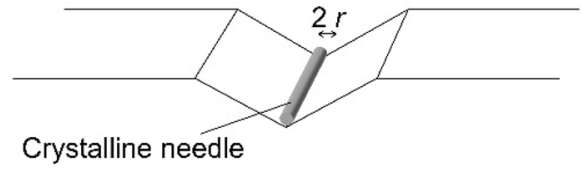

D

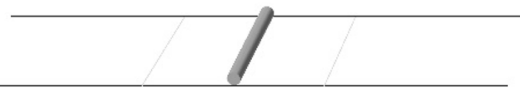

Fig. 7 Model for the formation of crystalline needles from the solution trapped in the creases 
typically $6 \mu \mathrm{m}$ thick (Fig. 5), which were approximately twice thicker than expected. Therefore, we infer that the solution will first flood over the crease (Fig. 7a). By the evaporation of the solvent, the solution is concentrated and trapped in the crease (Fig. 7b), followed by the formation of a crystalline needle (Fig. 7c). Finally, the crease disappears due to drying of PDMS (Fig. 7d).

As reported above, when IA, PA, MEK, and DGDE were used as the solvents, crystalline needles were formed on the sides of the thread (Fig. 5). However, when NMP and $\mathrm{N}, \mathrm{N}$-dimethylformamide (DMF), 3-ethoxypropionitrile (EPN), and acetonitrile (AN) were used, no crystalline needles were formed. As previously reported, these solvents can expand the oxidized PDMS surface and cause undulation due to the buckling instability [12]. The amplitude of the undulation was, however, much lower than that induced by IA and DGDE. The depths of the crease induced by DMF and NMP were only $\sim 1.6 \mu \mathrm{m}$ and $\sim 0.4 \mu \mathrm{m}$, respectively. Because crystalline needles are formed by drying of the solvent trapped in creases (Fig. 7), it is understandable that no crystalline needles are formed when DMF and NMP are used.

Such differences in dimensions of undulation between solvents like IA and solvents like DMF are understood as follows: In general, the periodicity $(\lambda)$ and amplitude $(A)$ of undulation are expressed by the following equations: $\lambda \sim\left(\frac{B}{K}\right)^{1 / 4}$

$A \sim \lambda\left(\frac{\Delta}{W}\right)^{1 / 2}$

where $B$ is the bending stiffness of the surface layer, $K$ is the effective stiffness of the foundation, and $(\Delta / W)$ is an imposed compressive strain [16]. These equations indicate that the softer the foundation the higher is the amplitude. When a strip of the PDMS is dipped in DGDE, IA, MEK, or PA, PDMS swells, thus softens. However, when dipped in NMP, DMF, EPN, or AN, PDMS does not swell. Therefore, it is suggested that the PDMS as the foundation was not softened by solvents like NMP; thus, the formed creases were small.

Furthermore, we attempted to determine the optimal composition of the solution for the regular arrangement of crystalline needles. A mixture of PA and ethyl acetate (EA) was used in several different ratios. The concentration of DBA was also varied. In addition, the experimental procedure was also improved, as described in detail in Sect. 2.6. When the solution was prepared by mixing $(a)$ pure EA and $(b)$ a saturated solution of DBA in PA $(a: b=3: 2, w / w)$, DBA crystalline needles were arranged regularly in some extent (Fig. 8). The regularity was improved compared
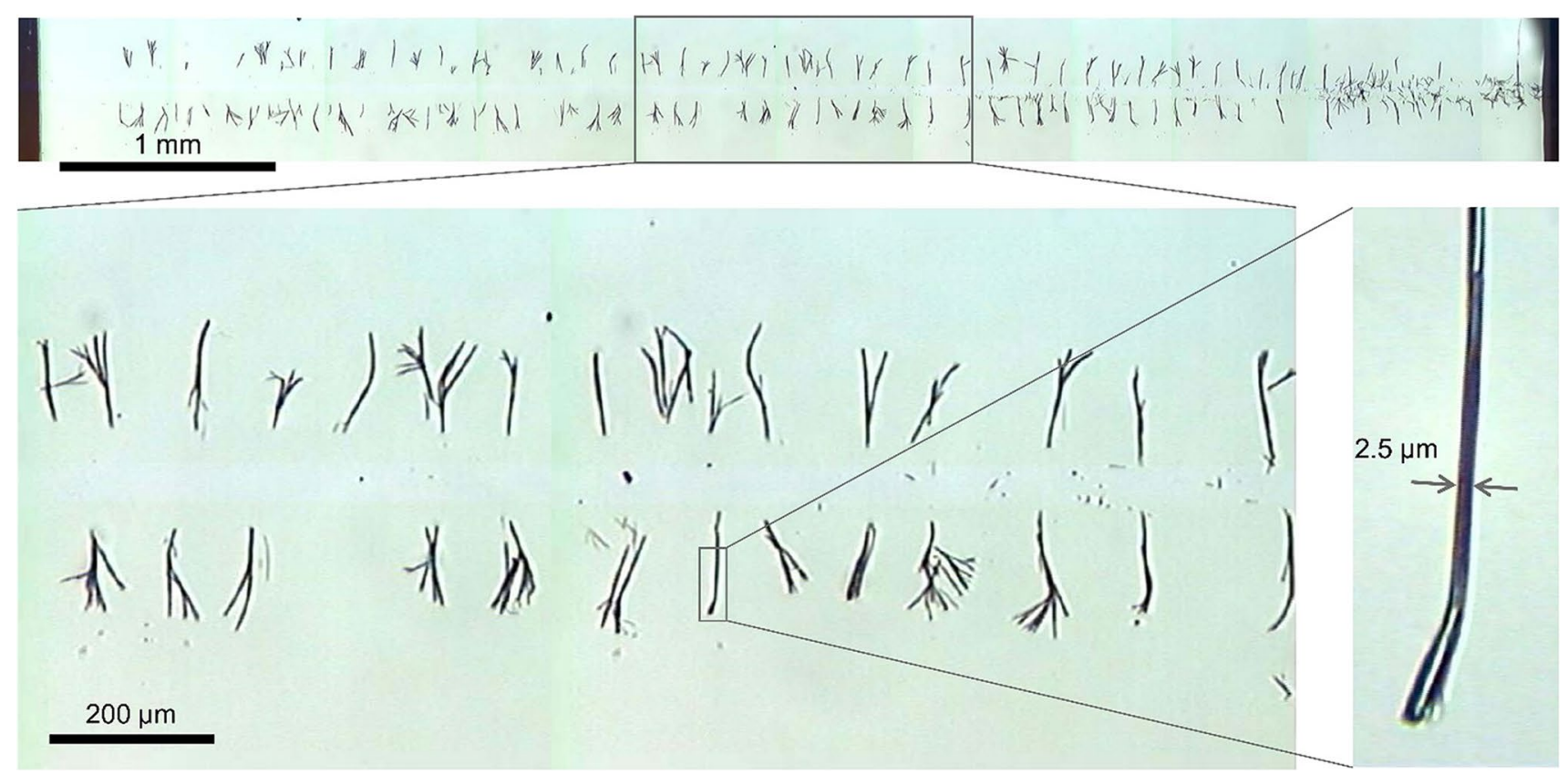

Fig. 8 DBA crystalline needles aligned using thread as a channel. The fluid was a mixture of pure ethyl acetate and a saturated solution of DBA in propyl acetate (3:2 w/w) (Experimental condition: No. 6) 
to that obtained from the saturated solution of DBA dissolved in IA (Fig. 5).

The orientation, length, and spacing of the crystalline needles in the microscope image (Fig. 8) were evaluated. Because most of the crystalline needles were branched, we fitted a sector to each needle and defined the orientation and length of the needle as the averaged orientation of the two sides and the length of the radius in the sector, respectively (Fig. $\mathrm{S} 6$ of the Electronic supplementary material). The spacing was defined as the lateral component of the distance between the vertexes of adjacent sectors.

As shown in Fig. 9a, the orientation of the crystalline needles was well controlled $\left(91 \pm 20^{\circ}\right)$. The length of the needles was $100 \pm 34 \mu \mathrm{m}$, indicating that the length distribution was broad (Fig. 9B). However, there were no needles longer than $156 \mu \mathrm{m}$, suggesting that the length was effectively limited. The needle spacing interval was not uniform (Fig. 9c). The microscope image (Fig. 8) suggests that often several needles were arranged in a single crease, resulting in nonuniform intervals. This is supported by the facts that the interval mode (i.e., the value that appears most often) was $120 \mu \mathrm{m}$ and that most intervals were shorter than $120 \mu \mathrm{m}$.

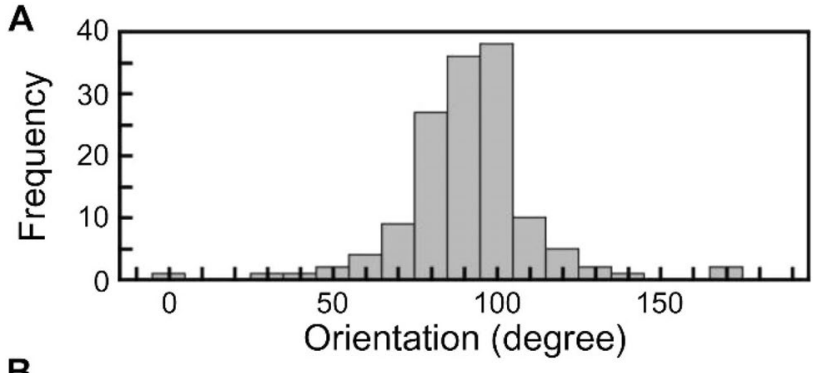

B
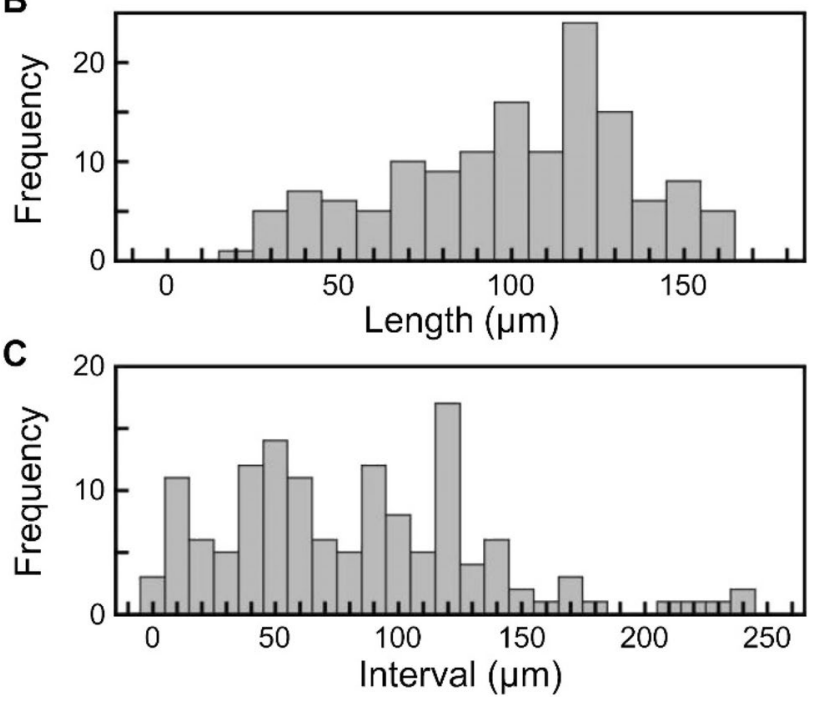

Fig. 9 Evaluation of the orientation, length, and spacing of the crystalline needles
The crystalline needles were also observed using an SEM. The orientation of the needles showed little variation. The SEM image also showed that the crystalline needles were clearly on the surface, not embedded in the PDMS. (Fig. 10)

The solvent and concentration of the DBA solution affected the number, thickness, and length of the crystalline needles. Solvents with higher boiling point (DEDG: 188-190 ${ }^{\circ} \mathrm{C}$; IA: $140^{\circ} \mathrm{C}$ ) tended to give longer crystalline needles than solvents with lower boiling point (PA: $102^{\circ} \mathrm{C}$; MEK: $80^{\circ} \mathrm{C}$ ), as shown in Fig. $\mathrm{S} 7$ in the Electronic supplementary material. For solvents with higher boiling point, the solvent remained in the creases for a longer period, which favored the growth of longer crystalline needles. As previously reported [12], solubility and concentration also affect crystalline growth in the creases. We are now planning systematic investigations to clarify factors affecting crystalline growth.

\section{Conclusions}

This study showed that thread can be used as microfluidic channels. The channels were fabricated by sandwiching the thread between two plates. The problem of fluid flooding into the space between the plates was solved by forming a reservoir designed to ensure that the total surface energy of the system was unchanged by the flooding. The channels could be filled with a wide variety of organic solvents including highly polar (e.g., NMP) and less polar (e.g., MEK) solvents. Satisfactory results were observed for both oil-repellent and hydrophilic plates.

When the plate was a PDMS-coated glass slide and its surface was oxidized, an organic semiconductor was

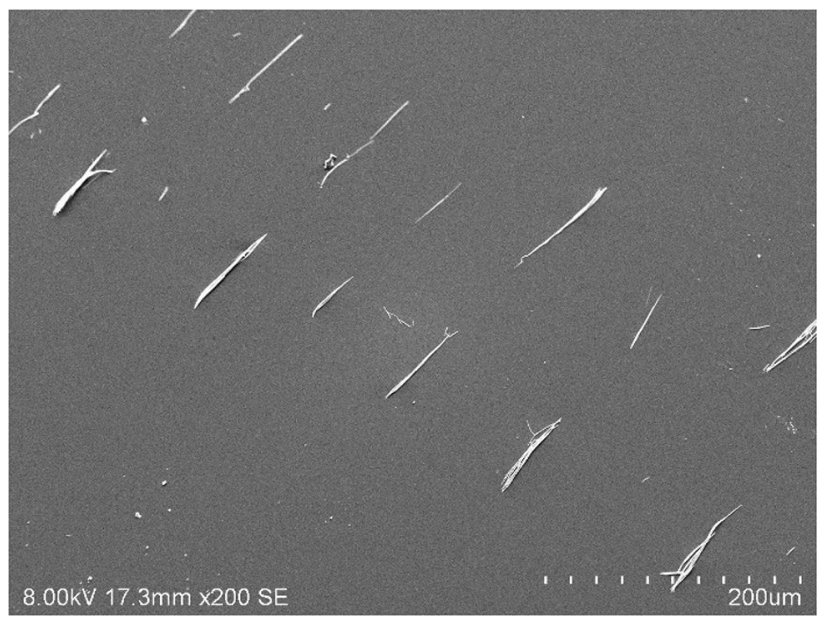

Fig. 10 SEM image of the aligned crystalline needles of 9,10-dibromoanthracene 
precipitated as aligned crystalline needles. This was due to the regular undulation of the PDMS surface induced by the organic solvents that filled the channel. This method is advantageous for handling fragile, thin crystalline needles because any external force was applied during the alignment. However, multiple crystalline needles often precipitated in a single crease, which caused nonuniform spacing of the needles. Experimental conditions, including solution concentration and evaporation speed, should be further investigated to overcome this disadvantage.

The crystalline needles were precipitated on the area outside of the channel. This is unique to the channels made of thread because with pipes that are normally used as microfluidic channels, only the space inside the channels can be used.

We are planning to perform measurements of the electronic properties (for example, conductivity) and characterization of the crystals (for example, single/polycrystal, degree of crystallinity) after the uniformity of spacing of the crystalline needles has been improved.

This facile method for regular arrangement of crystalline needles will be applied to the production of organic field-effect transistors in the future. During the production process, the crystalline needles will be transferred to substrates using a method similar to the transfer printing reported by Sun et al. [17].

Acknowledgements The authors appreciate Mr. Ryo Ishibashi and Mr. Shota Moteki in Shinshu University for their support provided in the experiments.

\section{Compliance with ethical standards}

Conflict of interest The authors declare that they have no conflict of interest.

\section{References}

1. Py C, Bastien R, Bico J, Roman B, Boudaoud A (2007) 3D aggregation of wet fibers. EPL 77:44005. https://doi. org/10.1209/0295-5075/77/44005

2. Safavieh R, Zhou GZ, Juncker D (2011) Microfluidics made of yarns and knots: from fundamental properties to simple networks and operations. Lab Chip 11:2618-2624. https://doi. org/10.1039/c1lc20336c

3. Farajikhah S, Cabot JM, Innis PC, Paull B, Wallace G (2019) Lifesaving threads: advances in textile-based analytical devices. ACS Comb Sci 21:229-240. https://doi.org/10.1021/acscombsci $.8 \mathrm{~b} 00126$
4. Weng X, Kang $Y$, Guo Q, Peng B, Jiang $H$ (2019) Recent advances in thread-based microfluidics for diagnostic applications. Biosens Bioelectron 132:171-185. https://doi.org/10.1016/j. bios.2019.03.009

5. Cabot JM, Breadmore MC, Paull B (2018) Thread based electrofluidic platform for direct metabolite analysis in complex samples. Anal Chim Acta 1000:283-292. https://doi.org/10.1016/j. aca.2017.10.029

6. Banerjee SS, Roychowdhury A, Taneja N, Janrao R, Khandare J, Paul D (2013) Chemical synthesis and sensing in inexpensive thread-based microdevices. Sens Actuators B 186:439-445. https://doi.org/10.1016/j.snb.2013.06.036

7. Erenas MM, de Orbe-Paya I, Capitan-Vallvey LF (2016) Surface modified thread-based microfluidic analytical device for selective potassium analysis. Anal Chem 88:5331-5337. https://doi. org/10.1021/acs.analchem.6b00633

8. Su B, Wu Y, Jiang $L$ (2012) The art of aligning one-dimensional (1D) nanostructures. Chem Soc Rev 41:7832-7856. https://doi. org/10.1039/c2cs35187k

9. Tong Y, Tang Q, Lemke HT, Moth-Poulsen K, Westerlund F, Hammershoj P, Bechgaard K, Hu W, Bjornholm T (2010) Solutionbased fabrication of single-crystalline arrays of organic nanowires. Langmuir 26:1130-1136. https://doi.org/10.1021/la902 $223 \mathrm{k}$

10. Tang $Q$, Jiang $L$, Tong $Y$, Li H, Liu YL, Wang ZH, Hu WP, Liu YQ, Zhu DB (2008) Micrometer- and nanometer-sized organic single-crystalline transistors. Adv Mater 20:2947-2951. https://doi. org/10.1002/adma.200800669

11. Huang Y, Duan X, Wei Q, Lieber CM (2001) Directed assembly of one-dimensional nanostructures into functional networks. Science 291:630-633. https://doi.org/10.1126/scien ce.291.5504.630

12. Watanabe $M$, Koga $Y$, Ichikawa $H$ (2019) Fabrication of regular arrays of organic crystalline needles using creases formed on oxidized poly(dimethylsiloxane) surfaces. J Appl Polym Sci 136:47736. https://doi.org/10.1002/app.47736

13. Washburn EW (1921) The dynamics of capillary flow. Phys Rev 17:273-283

14. Breid D, Crosby AJ (2009) Surface wrinkling behavior of finite circular plates. Soft Matter 5:425-431. https://doi.org/10.1039/ b807820c

15. Watanabe M (2012) Wrinkles with a well-ordered checkerboard pattern, created using dip-coating of poly(methyl methacrylate) on a UV-ozone-treated poly(dimethylsiloxane) substrate. Soft Matter 8:1563-1569. https://doi.org/10.1039/c2sm06669f

16. Cerda E, Mahadevan L (2003) Geometry and physics of wrinkling. Phys Rev Lett 90:074302. https://doi.org/10.1103/PhysR evLett.90.074302

17. Sun Y, Rogers JA (2004) Fabricating semiconductor nano/ microwires and transfer printing ordered arrays of them onto plastic substrates. Nano Lett 4:1953-1959. https://doi. org/10.1021/nl048835I

Publisher's Note Springer Nature remains neutral with regard to jurisdictional claims in published maps and institutional affiliations. 